\title{
Author Index Vol. 5, 1996
}

Abdul-Gaffar, S. 32 Abdul Sathar, S. 76 Abul,A.T. 19 Abul,H.T. 19 Abushaban, L. 137

Afifi,Z.E.M. 91 Akanji,A.O. 151 Al-Abdel, Y.K. 104 AlAnzi,A.A. 227 Al-Athary, E.A. 19 AlAwad, S.A. 114 AlAwadi,S. 234 Al-Awadi, S.A. 167 Al-Busairi, W. 167 Al-Ghanim, M. 167 Al-Haj,B. 114 Al-Mohannadi, S. 25 Al Mufti, S. 108 Al-Najdi, A.K. 104 Al-Nassar, K.E. 163 Al-Onazie,E. 104 Al-Othman, S. 121 Al-Rowaih, A. 32 Al-Saleh, Q.A. 114 Al-Sayed,A. 121 Al-Suleiman, A.S. 151 Al-Suweih, N. 101 Al-Zaabi,K. 25 Ali,J.H. 160 Arioglu,P.F. 12 Behbehani, A.E. 19

Cantekin, D. 12 Ceyhan,N. 187 Chadha,G. 47 Chadhi,G. 222 Cherian,G. 198 Christenson, J.T. 1 Dashti, H.M. 19 Dawson,K.P. 218

Dev,B.R. 129 Dhar,R. 104 Douglas, A.S. 97 D’Souza,B. 234 Dündar,S.V. 146

Endrys, J. 137

Farag,T.I. 114,118,167 Farah, S. 43, 108,114,

118 Ficicioglu, C. 12 Fido,A.A. 208

Galhotra, H.K. 86 Ghazala,B.S. 218 Gopalakrishnan, G. 160 Gurbuz, A. 12

Haider, S. 121 Hashad,M. 192 Hassan, M. 114 Hayat,N. 137,198 Haznedaroglu, í.C. 146

Henein, M.M. 47,222 Hira,P.R. 38 Humad,S.M. 227 Hussain, T. 19 Hussein, J.A. 43 Hussein, J.M. 108

Ismail, LA. 91

Jahan, S. 25 John,S.J. 129 Johny,K.V. 160 Juma,T.H. 86 Junaid,T.A. 192

Kandil,H. 167 Kannel,W.B. 172 Kavak,Z. 187

Kクallaf,F. 32 Khan,N. 198 Khan,R.A. 108 Khan,Z.U. 238 Khuraibit,A. 118 Kinatamitath, P. 151 Kirazh,S. 146 Kolk, B.A. van der 59

Lulu,A.R.D. 227 Luthra,U.K. 38

McDonald, G.A. 97 McFarlane, A.C. 59 Madda,J.P. 38 Mady,S.A. 167 Marafi, E. 104 Maurice, J. 1 Mavra,M. 47,222 Mokaddas, E.M. 238 Mubashir, M.A. 167 Murthy,S.K. 163 Mustapha, M.M. 97

Naefie, R. 38 Naem, M. 25 Nampoory, N.M. 160 Nayak,N. 38 Nayak,N.C. 76

Ownuwanne, A. 25 Özcebe, O.î. 146 Özdemir, O. 146

Patel,J.G. 238 Pekin,S. 187 Philip, L. 129 Pinto, R.G. 101 Pospula,W. 32

Qasrawi,B. 114, 167

Ravindran,M. 43, 108 Rice,P.L. 51 Rudwan,M.A. 108, 114

Sabry,M.A. 114,118,

167 Salama,A.L. 137,198 Samilchuk,E. 234 Sanyal,S.C. 238 Sarkar,C. 76 Sayinalp, N.M. 146 Schmuziger, M. 1 Shah, M.A. 121 Shah, N.M. 121 Shawis,R. 218 Shehadeh,F. 212 Sheikh, Z. 38 Shlokovich, S. 43 Shubaili, A.F. 118 Shuhaiber,H. 198 Simonet, F. 1 Singh, R.K.A. 86

Tahzeeb, S. 151 Tasdemir, S. 12 Tuli,M. 25

Unlu,R. 12 Usha,R. 167 Uthaman,B. 137, 198

Velebit,V. 1 Verghese, L. 163 Voevodin,A. 108 
Wahby, M.A.E. 212

Yacoub,N.H. 104

244 